Open Access

\title{
Effect of consuming novel foods consisting high oleic canola oil, barley $\beta$-glucan, and DHA on cardiovascular disease risk in humans: the CONFIDENCE (Canola Oil and Fibre with DHA Enhanced) study - protocol for a randomized controlled trial
}

Vanu R. Ramprasath ${ }^{1,2}$, Sijo J. Thandapilly ${ }^{1,2,3}$, Shuo Yang ${ }^{1,2}$, Anjalika Abraham ${ }^{1,2}$, Peter J. H. Jones ${ }^{1,2}$ and Nancy Ames ${ }^{1,2,3^{*}}$

\begin{abstract}
Background: Metabolic syndrome (MetS) has been identified as a major contributor to the development of cardiovascular disease (CVD). Current recommendations for dietary management of people with MetS involve quantitative and qualitative modifications of food intake, such as high consumption of vegetables, fruits, and whole grain foods. The results from our previous human trials revealed the potential of the dietary components high-oleic acid canola oil (HOCO)-docosahexaenoic acid (DHA) and high molecular weight barley $\beta$-glucan individually in managing CVD risk factors. Foods with a combination of HOCO-DHA and barley $\beta$-glucan have never been tested for their effects on CVD risk. The objective is to determine the effects of consuming novel foods HOCO-DHA, and barley $\beta$-glucan on managing CVD risk factors in people with MetS.

(Continued on next page)
\end{abstract}

\footnotetext{
* Correspondence: nancy.ames@agr.gc.ca

${ }^{1}$ Richardson Centre for Functional Foods and Nutraceuticals, University of

Manitoba, 196 Innovation Drive, Winnipeg, MB R3T 2N2, Canada

${ }^{2}$ Department of Human Nutritional Sciences, University of Manitoba,

Winnipeg, MB, Canada

Full list of author information is available at the end of the article
} 
(Continued from previous page)

Methods/Design: We are conducting a randomized, single-blind crossover trial with four treatment phases of 28 days each separated by a 4-week washout interval. Participants $(n=35)$ will be provided with weight-maintaining, healthy balanced diet recommendations according to their energy requirements during the intervention periods. Participants will receive muffins and cookies as treatment foods in a random order and will consume at least one meal per day at the research center under supervision. The four treatments include muffins and cookies consisting of (1) all-purpose flour and HOCO-DHA (50 g/day); (2) barley flour (4.36 g/day of $\beta$-glucan) and a blend of sunflower oil, safflower oil, and butter as control oil (50 g/day); (3) barley flour (4.36 g/day of $\beta$-glucan) and HOCO-DHA (50 g/ day; dosage of DHA would be $3 \mathrm{~g} /$ day); and (4) all-purpose flour and control oil (50 g/day). At the beginning and end of each phase, we will evaluate anthropometrics; systolic and diastolic blood pressure; blood lipid profile; low-density lipoprotein subfractions and particle size; 10-year Framingham CVD risk score; inflammatory status; and plasma and red blood cell fatty acid profiles, fecal microbiome, and body composition by dual-energy X-ray absorptiometry.

Conclusion: Cholesterol synthesis will also be studied, using a stable isotope approach. The proposed study will lead to innovation of novel food products, which may result in improvement in the overall cardiovascular health of humans.

Trial registration: Clinical trials.gov identifier: NCT02091583. Date of registration: 12 March 2014.

Keywords: Canola oil, Barley, $\beta$-glucan, DHA, Fiber, Cardiovascular disease, Metabolic syndrome, CONFIDENCE trial

\section{Background}

Metabolic syndrome (MetS), comprising a spectrum of chronic disease risk factors that include abdominal obesity, dyslipidemia, hypertension, and elevated fasting plasma glucose, has been identified as a major contributor to the development of cardiovascular disease (CVD) $[1,2]$. In North America, $34 \%$ of the adult population are affected by MetS, and MetS occurrence is even higher in other parts of the world [3]. Accordingly, MetS has become a significant public health concern and a burden on the global health care system, as this disorder is associated with increased morbidity, mortality, and related health care costs.

Current recommendations for dietary management in people with high risk for MetS involve quantitative and qualitative modifications in food intake, such as higher consumption of vegetables, fruits and whole grain food components with their inherent bioactive composition. More specifically, the presence of Mediterranean dietary components $[4,5]$, amounts of long chain $n-3$ fatty acids $[6,7]$, types and amounts of carbohydrates and dietary fiber content, are some dietary elements reported to show remarkable positive effects in the management of MetS. Important components include high-oleic acid canola oil (HOCO), barley $\beta$-glucan, docosahexaenoic acid (DHA).

Canola oil is derived from canola seed, which is obtained from a bright yellow flowering plant belonging to the Brassicaceae family [8]. Canola oil is considered the third-largest vegetable oil by sales volume after palm and soybean oil [9]. Canola's nutritional profile includes a low level $(7 \%)$ of saturated fatty acids; significant amounts of monounsaturated fatty acids (MUFAs) and polyunsaturated fatty acids (PUFAs), with $61 \%$ oleic acid, $21 \%$ linoleic acid, and $11 \% \alpha$-linolenic acid [10]; plant sterols (PSs) (0.53-0.97\%); and tocopherols (700$1200 \mathrm{ppm}$ ) [8]. Canola oil has proven efficacy in preventing and/or managing chronic ailments, including dyslipidemia, insulin resistance, and inflammation [11, 12]. As a result, in 2006, the U.S. Food and Drug Administration (FDA) approved a qualified health claim for canola oil suggesting that canola oil may help reduce the risk of CVD when substituted for saturated fat in the diet [8].

MUFAs, one of the components of canola oil, help reduce CVD risk by lowering blood pressure, blood total cholesterol (TC), and low-density lipoprotein cholesterol (LDL-C) levels; inhibiting LDL-C oxidation [13-16]; and improving glycemic control [17-19]. Oleic acid-enriched canola oil was developed to enhance the health benefits of canola oil related to CVD risk reduction. Its health benefits were compared with regular canola oil in humans in a randomized crossover human trial [12]. In that trial, consumption of HOCO for 28 days was shown to lead to reductions in TC and LDL-C levels and LDL-C/high-density lipoprotein cholesterol (HDLC) values, as compared with regular canola oil and a control diet without HOCO [12]. The results of a recently completed multicenter randomized controlled trial done at our laboratory validated these findings. In that study, the serum triglycerides (TGs) and 10-year Framingham risk scores, TC/HDL-C and TG/HDL-C ratios, and systolic blood pressure were significantly reduced, and HDL was increased, after consumption of HOCO-DHA compared with regular canola and control diets [20-23].

Consumption of fiber has been indicated to reduce the mortality rate in high-risk CVD populations [24]. $\beta$-glucan is a polysaccharide found mainly in yeast, mushrooms, 
oats, and barley in the form of fiber [25]. $\beta$-glucan fibers are located mainly in the endosperm cell walls of cereals. Among cereals, barley and oats are the richest sources of $\beta$-glucan, containing $4.0-7.0 \%$ and $2.2-7.8 \% \mathrm{wt} / \mathrm{wt}$ of $\beta$ glucan, respectively, compared with other cereals, such as rye and wheat [26]. Similarly to canola oil, $\beta$-glucan has gained significant attention among nutritional researchers because it has been shown to have potentially healthpromoting effects in animal and human studies [25, 27]. Consequently, in 1997, the FDA approved a health claim regarding $\beta$-glucan from whole oats for reducing the risk of heart disease [28, 29]. More recently, the European Food Safety Authority and Health Canada approved similar health claims for foods containing oats or barley $\beta$ glucan $[30,31]$.

In agreement with the previous studies, we also demonstrated the beneficial effects of high molecular weight $\beta$-glucan in healthy adults in a recent randomized, controlled-diet, crossover trial $(n=35)$. The results showed that consumption of $3 \mathrm{~g}$ or $5 \mathrm{~g}$ per day of high molecular weight barley $\beta$-glucan is highly effective in reducing TC and LDL cholesterol in adults with moderate hypercholesterolemia [32].

Long-chain PUFAs such as eicosapentaenoic acid (EPA) and DHA from fish and marine source oils, including algal oil, have commercially emerged as dietary supplements due to their health benefits. Long-chain fatty acids, including DHA, were shown to lower TG and increase serum HDL-C concentrations [33]. DHA is also capable of acting as an antiatherogenic factor by inhibiting inflammatory processes and thereby inhibiting plaque formation [34]. On the basis of the vast amount of knowledge accumulated over the years, the American Heart Association has recommended the use of n-3 fatty acids, including DHA, for secondary prevention of cardiovascular events in people with documented coronary artery disease.

Considering the fact that CVD is a multifactorial disease, the dietary approaches that combine different bioactives with enhanced health benefits have recently been well elucidated. For instance, a portfolio containing a low-fat vegetarian diet enriched by the inclusion of PSs, soy protein, viscous fiber, and tree nuts has been shown through our collaborations to markedly reduce LDL-C concentrations to an extent comparable in magnitude with reductions achieved using first-generation statins $[35,36]$. Likewise, a large body of research has demonstrated the synergetic effects attained by introducing two or more food bioactive components into the diet to achieve maximal health benefits through multimechanistic effects [37-39]. Accordingly, combining highly bioactive components such as HOCO, barley $\beta$-glucan, and DHA is expected to produce great synergistic and pleotropic beneficial effects on CVD risks factors compared with the individual biological actions listed above.

The overall objective of the present study is to test novel foods containing the most effective bioactives, including n-3 fatty acid-enriched dietary oil high in MUFAs and soluble dietary fiber, with aims of managing CVD risk factors in people with MetS and testing the efficacy and safety of these dietary components in humans.

\section{Methods/Design}

\section{Design of the human intervention trial}

A randomized, single-blind, crossover trial will be conducted at the Clinical Nutrition Research Unit at the Richardson Centre for Functional Foods and Nutraceuticals (RCFFN), University of Manitoba, Winnipeg, MB, Canada. The study design will consist of four phases with 28 days per phase, and each phase will be separated by 4 -week washout periods (Fig. 1). Participants will consume at least one of three daily meals under supervision, along with the treatment foods during weekdays from days 2 to 29 of each phase. Treatment muffins and cookies for the weekend will be packed and provided to participants to take home on Fridays. The other meals will be purchased by the participants from grocery stores on their own. Almost half of the treatments will be consumed under the supervision of a clinical coordinator to ensure optimal compliance. Participants will be instructed to consume no alcoholic or caffeinated beverages. Participants will be instructed not to consume more than one serving of fish or seafood products per month during the study. A 3-day food record will be collected before the study and after each treatment phase. Participants will be strongly recommended to maintain consistency in their physical activities during the experimental period. Compliance with the treatment products will be determined from checklists and by measuring the amount of leftover food if any in the containers returned by the participants the following day.

Diets will be planned for every participant according to individual energy requirements and will be nutritionally adequate. Basal energy requirement will be estimated using the Mifflin-St Jeor equation [40]. The basal energy requirements will be multiplied by an activity factor of 1.7 to supply the additional energy needs of mildly to moderately active healthy adults. During the study period, body weight will be monitored. If any of the participants gain or lose weight during the first week, adjustments to their estimated energy requirements will be made. Participants will be given recommendations to follow a typical Western diet that meets the Canadian Dietary reference intake. The nutrient content of the participant's diet, based on the 3-day food record, will be analyzed using Food Processor Nutrition Analysis 


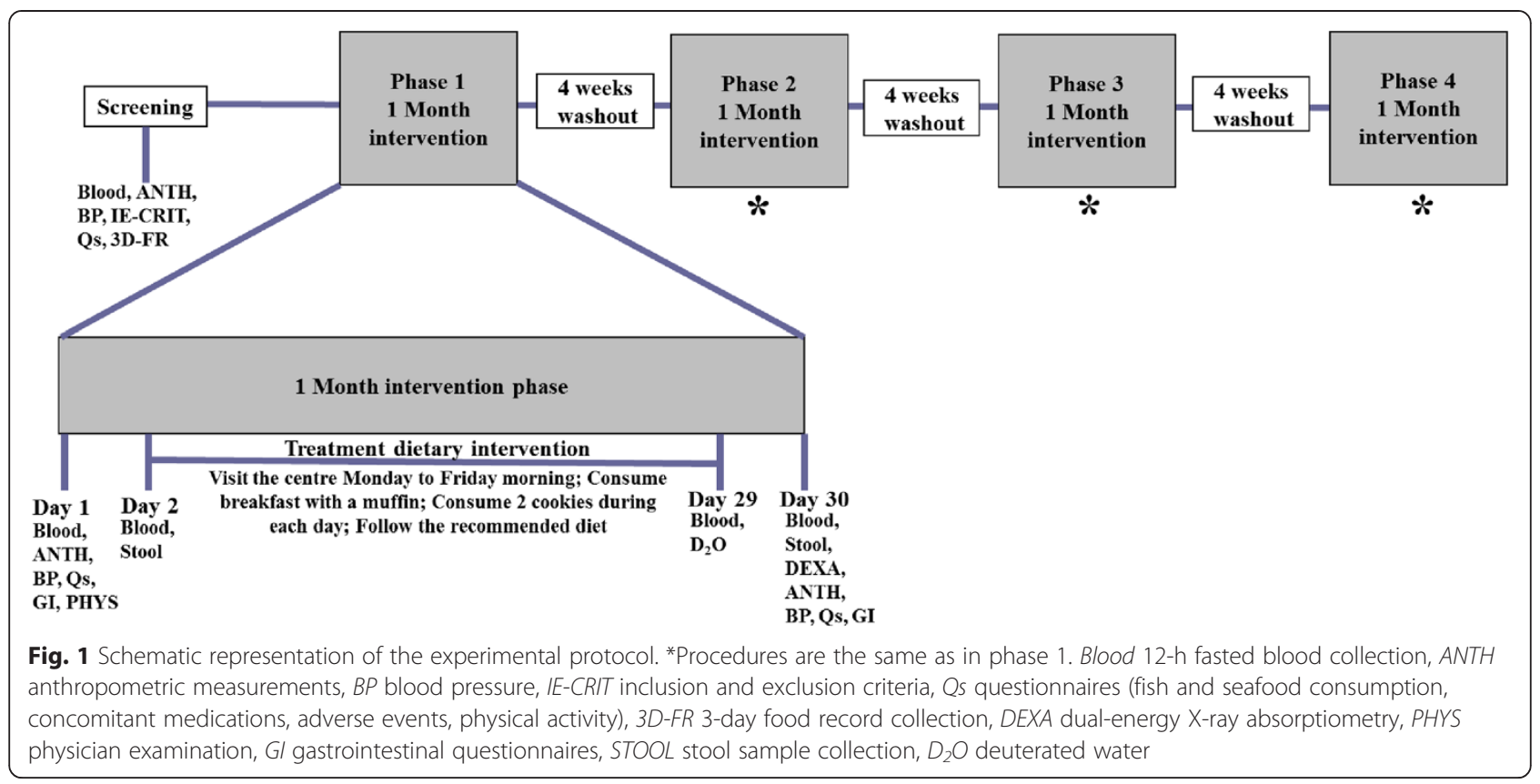

software (ESHA Research, Salem, OR, USA) to ensure the intake of macro- and micronutrients and compliance with the protocol. Treatments will be isocalorically incorporated into muffins and cookies and consumed at breakfast, in the evening, as snacks, and with supper. Recruited participants will be randomly assigned to one of the treatment sequences using a Latin square design. Participants will be required to consume one muffin along with their breakfast and two cookies during their evening snack and dinner, respectively.

The four treatments are as follows: (1) muffins and cookies containing all-purpose flour and $50 \mathrm{~g} /$ day HOCO-DHA ( $85 \% / 15 \%$; DHA oil consists of $40 \%$ DHA) (DHA dosage $3 \mathrm{~g} /$ day); (2) muffins and cookies containing barley flour with $4.36 \mathrm{~g} /$ day of high molecular weight barley $\beta$-glucan and $50 \mathrm{~g} /$ day of a blend of sunflower oil, safflower oil, and butter as a control oil; (3) muffins and cookies containing a combination of barley flour with $4.36 \mathrm{~g} /$ day, high molecular weight barley $\beta$ glucan, and $50 \mathrm{~g} /$ day of HOCO-DHA; and (4) muffins and cookies containing all-purpose flour and $50 \mathrm{~g} /$ day of control oil. The control oil used in the study represents a typical Western diet fat intake as a control treatment composed largely of saturated fat with substantial levels of n-6 linoleic acid common in current North American dietary intakes. Dosages for HOCO-DHA and barley $\beta$ glucan were determined on the basis of previous clinical studies done by our research group [20, 21, 32]. Muffin and cookie recipes were developed in Agriculture and Agri-Food Canada laboratories at the RCFFN metabolic kitchen. Barley grain (cultivar CDC Rattan) was provided by the Alberta Barley Commission and milled into whole grain flour by the Canadian International Grains Institute. All-purpose flour was obtained from local supermarket. HOCO was provided by Richardson Oilseed Limited. life's DHA oil was purchased from DSM Nutritional Products (Ayr, ON, Canada). Macronutrients, including protein, carbohydrates, and fiber content of the foods, were analyzed at the Agriculture and Agri-Food Canada laboratories, RCFFN, Winnipeg, Canada. Total fat and fatty acid profiles of the foods were measured at the RCFFN laboratories (Winnipeg, MB, Canada). The muffin flavors include vanilla and spice, and the cookies were made in lemon, ginger, and chocolate flavors. The flavors of the muffins and cookies will be provided in rotation with equal numbers of days per flavor during each treatment period. The nutrient compositions of the muffins and cookies for the different treatments of the study are depicted in Tables 1 and 2, respectively. The macronutrient contents of treatment foods will be similar between different treatments, and also there are no differences in nutritional values between different flavors of muffins or cookies.

\section{Participant selection}

Thirty-five male and female participants with MetS will be enrolled in the study. They will be recruited using advertisements in local media, including newspapers, radio, and the RCFFN official website, as well as on university campuses. We will also recruit from our preexisting database of participants. As well, to present information about the nature of the study and procedures, sessions will be held at the RCFFN during the recruitment period, and potential study participants will be invited to 
Table 1 Details and nutritional composition of the study muffins

\begin{tabular}{|c|c|c|c|c|}
\hline Nutritional component measurements & Control flour + HOCO-DHA & Barley flour + control oil & Barley flour + HOCO-DHA & Control flour + control oil \\
\hline Weight (g) & 113 & 108 & 109 & 112 \\
\hline Energy $(\mathrm{kcal} / \mathrm{kJ})^{\mathrm{a}}$ & $379 / 1586$ & $383 / 1602$ & $383 / 1602$ & $379 / 1586$ \\
\hline Total carbohydrates $(\mathrm{g})^{\mathrm{a}}$ & 33 & 36.59 & 36.59 & 32.65 \\
\hline Total proteins $(g)^{a}$ & 8 & 9 & 9 & 8 \\
\hline Total fiber $(g)^{a}$ & 0.36 & 5.77 & 5.77 & 0.36 \\
\hline$\beta$-glucan $(g)^{a}$ & 0 & 2.18 & 2.18 & 0 \\
\hline Total fat (\% weight) & 24 & 23 & 23 & 23 \\
\hline SFA (\%) & 10.42 & 35.36 & 11.24 & 33.34 \\
\hline MUFA (\%) & 66.21 & 28.38 & 64.03 & 31.05 \\
\hline PUFA (\%) & 23.37 & 36.25 & 24.73 & 35.61 \\
\hline n-3 PUFA (\%) & 6.57 & 0.66 & 6.45 & 0.49 \\
\hline n-6 PUFA (\%) & 16.80 & 35.60 & 18.28 & 35.12 \\
\hline
\end{tabular}

DHA docosahexaenoic acid, HOCO high-oleic acid canola oil, MUFA monounsaturated fatty acid, PUFA polyunsaturated fatty acid, SFA saturated fatty acid ${ }^{a}$ Values were calculated based on values of the ingredients. Muffins were made with two different flavors: vanilla and spice. Values presented are the average of both the flavors, and there were no major differences between flavors for the measurements

these sessions. Potential participants will undergo a screening where blood pressure and anthropometric measurements will be performed and 10-ml fasting blood samples will be collected to test for lipid profiles. Eligible participants will be asked to provide written informed consent to participate in the study. The trial was approved by the biomedical research ethics committee at the University of Manitoba (B2014:029).

\section{Inclusion criteria}

Participants aged 18-70 years will be recruited. The participants should be slightly overweight with a body mass index $>25 \mathrm{~kg} / \mathrm{m}^{2}$ and waist circumference $>94 \mathrm{~cm}$ for men and $>80 \mathrm{~cm}$ for women in accordance with the
International Diabetic Federation MetS criteria for waist circumference. In addition, they have to be deemed to be otherwise healthy by the study physician. Additionally, participants should also meet at least two of the following criteria: (1) TG $>1.7 \mathrm{mmol} /$ $\mathrm{L}$, (2) HDL-C $<1 \mathrm{mmol} / \mathrm{L}$ for males and $<1.3 \mathrm{mmol} / \mathrm{L}$ for females, (3) fasting glucose $>5.6 \mathrm{mmol} / \mathrm{L}$, (4) LDL$\mathrm{C}>2.7 \mathrm{mmol} / \mathrm{L}$, and (5) blood pressure $>130 \mathrm{mmHg}$ for systolic and $>85 \mathrm{mmHg}$ for diastolic.

\section{Exclusion criteria}

Participants taking lipid-lowering medications or nutritional supplements known to affect blood lipids, or who have any dietary restrictions that would prevent them

Table 2 Details and nutritional composition of the study cookies

\begin{tabular}{|c|c|c|c|c|}
\hline Nutritional component measurements & Control flour + HOCO-DHA & Barley flour + control oil & Barley flour + HOCO-DHA & Control flour + control oil \\
\hline Weight (g) & 52 & 50 & 52 & 53 \\
\hline Energy $(\mathrm{kcal} / \mathrm{kJ})^{\mathrm{a}}$ & 213/891 & $221 / 924$ & $221 / 924$ & 213/891 \\
\hline Total carbohydrates $(\mathrm{g})^{\mathrm{a}}$ & 23 & 25 & 25 & 23 \\
\hline Total proteins $(g)^{a}$ & 4 & 4 & 4 & 4 \\
\hline Total fiber $(g)^{a}$ & 0.46 & 3.17 & 3.17 & 0.46 \\
\hline Beta glucan $(g)^{a}$ & 0 & 1.09 & 1.09 & 0 \\
\hline Total fat (\% weight) & 24 & 24 & 23 & 24 \\
\hline SFA (\%) & 12.50 & 35.39 & 12.58 & 33.75 \\
\hline MUFA (\%) & 63.37 & 28.95 & 64.23 & 31.08 \\
\hline PUFA (\%) & 24.13 & 35.67 & 23.20 & 35.16 \\
\hline n-3 PUFA (\%) & 6.44 & 0.61 & 6.28 & 0.48 \\
\hline n-6 PUFA (\%) & 17.53 & 34.91 & 16.91 & 34.69 \\
\hline
\end{tabular}

DHA docosahexaenoic acid, HOCO high-oleic acid canola oil, MUFA monounsaturated fatty acid, PUFA polyunsaturated fatty acid, SFA saturated fatty acid ${ }^{a}$ Values were calculated based on the values of the ingredients. Cookies were made with three different flavors: cocoa, ginger, and lemon. Values presented are the average of the flavors, and there were no major differences between flavors for the measurements 
from consuming the study diet for 28 days during each phase, will be excluded. Participants with a current or past history of any diseases and disorders that could interfere with fat absorption will be excluded. Individuals with hypertension having systolic blood pressure $>150 \mathrm{mmHg}$ or diastolic blood pressure $>100 \mathrm{mmHg}$ will be excluded from the study. Participants planning to become pregnant during the study period will be excluded. Smokers and people consuming more than one alcoholic drink/day or who have a history of alcoholism or drug dependence cannot be included in the study. Use of any experimental medication within 1 month before screening or as concomitant medication is also an exclusion criterion.

\section{Sample collection}

Twelve-hour fasting blood samples will be collected on days 1, 2, 29, and 30 of each intervention phase. Participants will be advised to be fasted for $12 \mathrm{~h}$ and not to consume any alcoholic beverage for at least $48 \mathrm{~h}$ before blood collection. Participants will also be instructed to refrain from engaging in intense physical activity (aerobic, spinning, and running) for at least $24 \mathrm{~h}$ before each visit. On days of blood collection, a checklist with questions on 12-h diet, 48-h alcohol, and 24-h physical activity will be administered and data recorded to determine participant compliance.

Blood samples will be centrifuged at $3000 \mathrm{rpm}$ for 20 minutes at $4{ }^{\circ} \mathrm{C}$; aliquoted to yield serum, plasma, and red blood cells (RBC); and then stored at $-80{ }^{\circ} \mathrm{C}$ until analyses. To obtain the plasma lipid profile, average values of days 1 and 2 will be considered as baseline and average values of days 29 and 30 will be considered as the endpoint of each treatment phase. In addition, the influence of these diets on gastrointestinal microbial diversity in the current study population will be measured. For this analysis, stool samples (four or five scoops totaling $4 \mathrm{~g}$ ) will be collected before and after each intervention phase (days 2 and 30) of the trial. Stool sample collection kits, including containers, will be provided for the participants to collect their stool samples. However, it is optional for the participants to provide the stool samples, and they may still participate if they choose not to provide stool samples.

\section{Stable isotope tracer intake}

To assess cholesterol fractional synthesis rate (FSR), participants will be asked to consume deuterated water $\left(\mathrm{D}_{2} \mathrm{O}\right)$ at the end of each phase. On day 29, $0.7 \mathrm{~g}$ of $\mathrm{D}_{2} \mathrm{O}$ per kilogram of estimated body water will be given orally before breakfast as a tracer to measure cholesterol FSR over $24 \mathrm{~h}$. Fasting blood will be obtained on days 29 and 30 .

\section{Clinical data collection}

Whole-body dual-energy X-ray absorptiometry (DEXA) (Prodigy Advance; GE Healthcare Lunar, Madison, WI, USA) will be conducted to determine changes in body fat composition during the last week of each phase. Blood pressure, body weight, and waist circumference will be monitored at the beginning and end of each treatment period. An automated blood pressure device will be used to measure blood pressure. Participants will be advised to rest quietly throughout the measurements. Blood pressure will be measured four times at 2-minute intervals. The first measurement will be ignored, and the last three measurements will be averaged to determine systolic and diastolic blood pressures. Gastrointestinal tolerability questionnaires will be completed by participants at the beginning and end of each intervention period. The 10-year Framingham CVD risk score will be calculated for each participant during each phase [41].

\section{Analytical methodology}

Plasma samples will be analyzed for total lipid profiles, including TC, HDL-C, and TG levels, as well as for glucose levels, using the automated enzymatic methods on a VITROS 350 Chemistry System (Ortho Clinical Diagnostics, Markham, ON, Canada). LDL-C values will be calculated using the Friedewald equation [42]. Subfractions and particle sizes of LDL-C after each treatment intervention will be determined by the proprietary Lipoprint Lipoprotein Subfractions Testing System (Quantimetrix, Redondo Beach, CA, USA).

Inflammatory markers such as plasma C-reactive protein and serum amyloid $\mathrm{A}$ levels will be measured with commercially available enzyme-linked immunosorbent assay (ELISA) kits. Plasma cytokines, specifically interleukin (IL)-1, IL-6, IL-8, IL-10, and tumor necrosis factor- $\alpha$, will be measured by multiplex human ELISA. Plasma soluble adhesion molecules such as soluble vascular cell adhesion molecule 1 , soluble intercellular adhesion molecule 1 , and soluble P-selectin and Eselectin will also be measured with commercially available human ELISA kits. Apolipoproteins A1, B, and E will be analyzed with commercially available ELISA kits.

Fasting plasma insulin concentrations will be determined using an ELISA kit. Insulin homeostasis modeling assessment will be used as an estimate for percentage $\beta$ cell function and insulin resistance.

\section{Plasma and red blood cell fatty acid analyses}

Plasma and RBC total lipids will be extracted using the Folch method [43], involving chloroform-methanol (2:1 
$\mathrm{vol} / \mathrm{vol}$ ) containing $0.01 \%$ 3,5-di-tert-4-butylhydroxytoluene (Sigma-Aldrich, Oakville, ON, Canada) and heptadecanoic acid as an internal standard (Sigma-Aldrich). Extracted fatty acids will be methylated with methanolic $\mathrm{HCl}$. Fatty acid methyl esters will be separated on a SUPELCOWAX 10 capillary gas chromatography column $(30 \mathrm{~m} \times 0.25 \mathrm{~mm}$ with $0.25-\mathrm{mm}$ film thickness; Supelco/Sigma-Aldrich, Bellefonte, PA, USA) using a gas chromatograph equipped with a flame ionization detector (Bruker 430; Bruker Daltonics, Billerica, MA, USA). Individual fatty acids will be identified by comparison with known standards (Nu-Chek Prep, Elysian, MN, USA). Individual fatty acid levels will be calculated according to the peak area relative to the total area and expressed as a percentage of total fatty acids.

Concentrations of isotope-labeled cholesterol in circulatory cholesterol will be measured over $24 \mathrm{~h}$. In this study, we will measure the deuterium enrichment into RBC cholesterol to calculate FSR of whole-body cholesterol. The initial 24-h interval of $\mathrm{D}_{2} \mathrm{O}$ uptake is considered to be the optimal period for measuring synthesis [44]. The analytical methods for isotope analyses are as follows. For determining cholesterol FSR (\%/day), RBC from day 29 and 30 will be saponified with freshly prepared $\mathrm{KOH}$-methanol at $100{ }^{\circ} \mathrm{C}$ for $1 \mathrm{~h}$, and the sterol fraction will be extracted with petroleum diethyl ether. RBC cholesterol deuterium enrichment will be quantified using a gas chromatography-pyrolysisisotope ratio mass spectrometry (Delta V Pulse Isotope Ratio Mass Spectrometer; Thermo Fisher Scientific, Bremen, Germany). Hydrogen gas reduced from water will be analyzed for deuterium enrichment against Vienna Standard Mean Ocean Water [45].

\section{Microbiome analysis}

Bacterial DNA from the fecal samples will be extracted using the ZR Fecal DNA MiniPrep kit (Zymo Research, Irvine, CA, USA), and DNA concentration and quality will be determined using a NanoDrop 2000c spectrophotometer (NanoDrop Products/ Thermo Fisher Scientific, Wilmington, DE, USA). Gut microbial composition will be analyzed by nextgeneration Illumina-based sequencing (Illumina, San Diego, CA, USA). Briefly, the V3-V4 region of the $16 \mathrm{~S}$ rRNA gene will be targeted for PCR amplification as described by Fadrosh et al. [46]. PCR products will then be purified using ZR-96 DNA Cleanup Kit (Zymo Research) and added to the MiSeq Reagent Kit V3 (300 cycles; Illumina) for sequencing reactions on a MiSeq platform. The paired-end Illumina fastq files will then be analyzed using the QIIME software package [47]. Principal coordinate analysis will be performed to evaluate the distance matrices by generating two-dimensional plots using PRIMER v6 software (PRIMER-E, Ivybridge, UK).

\section{Sample size calculation and statistical analysis}

Sample size was determined to detect an anticipated difference in LDL-C level of $7.4 \%(0.43 \mathrm{mmol} / \mathrm{L})$ due to HOCO consumption, compared with control, with a standard deviation estimate of $0.13 \mathrm{mmol} / \mathrm{L}$. The $\alpha$ and power were taken as 0.05 and 0.8 , respectively. A sample size of 35 participants was therefore calculated, with a target of 28 participants, taking into account the block size and the estimated $20 \%$ premature withdrawal rate of participants from the protocol, given the existing level of difficulty in completing the protocol elements.

Statistical analysis will be performed using SAS statistical software (SAS Institute, Cary, NC, USA). Baseline and endpoint measurements will be compared using the analysis of variance (ANOVA) model for determination of treatment effects. The results will be expressed as means with standard errors. The normality of distribution of data will be determined with the Shapiro-Wilk test, and the non-normal variables will be normalized before comparison with other treatment by log transformation. The effects of dietary treatments will be examined using mixed-model ANOVA with diet, sequence, phase, and center as fixed factors and subject as a random factor within the model. Statistical significance will be set at $p<0.05$ for all analyses. Significance between different dietary effects will be examined with the Bonferroni adjustment for multiple comparisons.

\section{Adverse events and concomitant medications}

No adverse events due to the study treatments are expected, based on the previous studies [20] and because the experimental components are foods. Adverse events, if any, will be monitored and will be reported in the case report form. All serious adverse events will be reported to sponsors and the institutional ethics board of the University of Manitoba. Serious adverse events will be brought to the attention of the study physician immediately for assignment of attribution. All prescription and over-the-counter medications either used at the start of the trial or initiated during the trial will be recorded.

\section{Conditions for withdrawal from the study}

The following events are to be considered sufficient reason for discontinuing a participant from the study: poor compliance (consuming $<70 \%$ of the given treatment foods and/or dietary guidelines) or noncompliance by the participant; developing condition(s) or consumption of fish, supplements, or medications as specified under the exclusion criteria; and presenting with an adverse event or any other medical 
situation where continuation of the study's treatment would compromise the participant's health.

\section{Confidentiality of data}

All the data collected for this study will remain confidential in accordance with the Personal Health Information Act of Manitoba, as applicable. However, the clinical research team members involved will know the identity of the participants. The researchers may know the identity of the participants if they are requested to respond to questions during the informed consent process. All research team members will respect the concept of confidentiality at all times.

Each participant will be assigned a unique code so that all personal information, including questionnaire information, will be confidential. Full names and other identifying information will not be revealed, unless required by law and/or for the applicable research ethics board review or for auditing purposes. The identity of the participants will not be revealed in any published data or in presentation of the information obtained as a result of this study. Medical records containing the identity of the participant will be treated as confidential.

\section{Data handling and storage}

All data collected will be collated and secured in a locked filing cabinet in a locked office at the RCFFN and made available only to the study investigators and study coordinators for research purposes. The data will include patient history information, which will be recorded when enrolling the participants in the trial. The purpose of accessing the data will be restricted to creation of final results tables and statistical interpretation. The data will not be used for any other purposes.

\section{Participant feedback}

The primary outcomes, including the 10-year Framingham CVD risk score and blood lipid profile results, will be given to the study participants when the analyses are completed. Participants will receive a sealed and confidential letter that states their individual results for the parameters measured for study purposes, along with the mean values obtained for the entire study population. The letter will be sent by the principal investigator at the RCFFN to the mailing address listed on the personal information form that participants fill out before enrollment in the study.

\section{Discussion}

Considering the huge health impact of dietary portfolio intervention approaches as highly efficient nutritional strategies for the modification of CVD risk factors, combination of food components and bioactives are getting tremendous attention as an alternative strategy to manage CVD risk factors. We anticipate that, taken together, the foods containing both HOCO-DHA and barley-derived high molecular weight $\beta$-glucan will possess a superior biological action due to their differential mechanistic effects, compared with the individual components, against CVD risk factors.

In the current proposal, combining these potent groups of food bioactives, such as HOCO-DHA and $\beta$-glucan, to achieve parallel multiplication of biological action and to reduce the CVD events substantially is of paramount clinical significance. This study will be the first investigation of the cholesterollowering effect of a combination of HOCO-DHA and barley $\beta$-glucan. The proposed study will help to improve understanding of the metabolic mechanisms underpinning the health benefits associated with consumption of foods consisting of HOCO-DHA and barley $\beta$-glucan. The proposed research will not only support the improvement of the cardiovascular health of Canadians but also increase the marketability of our canola-barley products internationally. The anticipated outcomes of the proposed study are expected to have a high probability of impacting the health and wellness of Canadians because (1) a significant proportion of the population has MetS and (2) scientific data generated by well-designed, unbiased human clinical trials are required to properly substantiate health effects of a combination of HOCO-DHA and barley $\beta$-glucan.

\section{Trial status}

The trial is registered at ClinicalTrials.gov (NCT02091583). Muffins and cookies required for the study have been developed and analyzed for their nutritional content. The clinical intervention of the study was initiated in January 2015, and recruitment and randomization of participants, along with the study dietary interventions, are ongoing.

\footnotetext{
Abbreviations

ANOVA: Analysis of variance; ANTH: Anthropometric measurements; BP: Blood pressure; CVD: Cardiovascular disease; DEXA: Dual-energy X-ray absorptiometry; 3D-FR: 3-day food record collection; DHA: Docosahexaenoic acid; $\mathrm{D}_{2} \mathrm{O}$ : Deuterated water; ELISA: Enzyme-linked immunosorbent assay; EPA: Eicosapentaenoic acid; FDA: U.S. Food and Drug Administration; Gl: Gastrointestinal questionnaires; FSR: Fractional synthesis rate; HDL-C: High-density lipoprotein cholesterol; HOCO: High-oleic acid canola oil; IECRIT: Inclusion and exclusion criteria; IL: Interleukin; LDL-C: Low-density lipoprotein cholesterol; MetS: Metabolic syndrome; MUFA: Monounsaturated fatty acid; PS: Plant sterol; PUFA: Polyunsaturated fatty acid; Qs: Questionnaires (fish and sea food consumption, concomitant medications, adverse events, physical activity); RBC: Red blood cells; RCFFN: Richardson Centre for Functional Foods and Nutraceuticals; SFA: Saturated fatty acid; TC: Total cholesterol; TG: Triglyceride.
} 


\section{Authors' contributions}

VRR, SJT, PJHJ, and NA hold the responsibility for the concept and design of the study. NA developed the foods for the trial, and her group performed food analysis for carbohydrates, proteins, and fiber. SY and AA have been making the muffins and cookies needed for the trial. SY and VRR performed the fat content and fatty acid profile analysis of the foods. SY and AA have been conducting the clinical intervention trial and acquiring data. VRR wrote the manuscript and is supervising the clinical study. All the authors reviewed and contributed to the manuscript, and all authors read and approved the final manuscript.

\section{Acknowledgments}

The trial is financially supported by the Canadian Institutes of Health Research (CIHR) Open Operating Grant Program (grant 312802). The authors acknowledge the Alberta Barley Commission for providing the barley grain. The Canadian International Grains Institute is acknowledged for milling the barley grain to whole grain flour. The authors are also thankful to the Richardson Oilseed Limited for providing the HOCO. The funding support for the study provided by the CIHR is also highly acknowledged. We thank Camille Rhymer, Tracy Exley, and Kim Kuzminski, Agriculture and Agri-Food Canada, for their help with food development and nutrient analysis of the study foods.

\section{Author details}

${ }^{1}$ Richardson Centre for Functional Foods and Nutraceuticals, University of Manitoba, 196 Innovation Drive, Winnipeg, MB R3T 2N2, Canada. ${ }^{2}$ Department of Human Nutritional Sciences, University of Manitoba, Winnipeg, MB, Canada. ${ }^{3}$ Agriculture and Agri-Food Canada, Winnipeg, MB, Canada.

Received: 18 May 2015 Accepted: 16 October 2015

\section{Published online: 31 October 2015}

\section{References}

1. Mozumdar A, Liguori G. Persistent increase of prevalence of metabolic syndrome among U.S. adults: NHANES III to NHANES 1999-2006. Diabetes Care. 2011:34(1):216-9. doi:10.2337/dc10-0879.

2. Paoletti R, Bolego C, Poli A, Cignarella A. Metabolic syndrome, inflammation and atherosclerosis. Vasc Health Risk Manag. 2006;2(2):145-52.

3. Al-Sarraj T, Saadi H, Volek JS, Fernandez ML. Metabolic syndrome prevalence, dietary intake, and cardiovascular risk profile among overweight and obese adults 18-50 years old from the United Arab Emirates. Metab Syndr Relat Disord. 2010;8(1):39-46.

4. Kesse-Guyot E, Ahluwalia N, Lassale C, Hercberg S, Fezeu L, Lairon D. Adherence to Mediterranean diet reduces the risk of metabolic syndrome: a 6-year prospective study. Nutr Metab Cardiovasc Dis. 2013;23(7):677-83.

5. Babio N, Toledo E, Estruch R, Ros E, Martínez-González MA, Castañer O, et al. Mediterranean diets and metabolic syndrome status in the PREDIMED randomized trial. CMAJ. 2014;186(17):E649-57. doi:10.1503/cmaj.140764.

6. Lopez-Huertas E. The effect of EPA and DHA on metabolic syndrome patients: a systematic review of randomised controlled trials. Br J Nutr. 2012;107 Suppl 2:S185-94.

7. Navas-Carretero S, Pérez-Granados AM, Schoppen S, Vaquero MP. An oily fish diet increases insulin sensitivity compared to a red meat diet in young iron-deficient women. Br J Nutr. 2009;102(4):546-53.

8. Lin L, Allemekinders H, Dansby A, Campbell L, Durance-Tod S, Berger A, et al. Evidence of health benefits of canola oil. Nutr Rev. 2013;71(6):370-85.

9. Wittenberger K. ERS/USDA Briefing Room - soybeans and oil crops: canola seed, oil, and meal. Washington, DC: U.S. Department of Agriculture, Economic Research Service. http://www.ers.usda.gov/topics/crops/soybeansoil-crops/canola.aspx. Last updated 10 Oct 2012; accessed 22 Oct 2015.

10. Johnson GH, Keast DR, Kris-Etherton PM. Dietary modeling shows that the substitution of canola oil for fats commonly used in the United States would increase compliance with dietary recommendations for fatty acids. J Am Diet Assoc. 2007;107(10):1726-34.

11. Iggman D, Gustafsson IB, Berglund L, Vessby B, Marckmann P, Risérus U. Replacing dairy fat with rapeseed oil causes rapid improvement of hyperlipidaemia: a randomized controlled study. J Intern Med. 2011;270(4):356-64. doi:10.1111/j.1365-2796.2011.02383.x.

12. Gillingham LG, Gustafson JA, Han SY, Jassal DS, Jones PJ. High-oleic rapeseed (canola) and flaxseed oils modulate serum lipids and inflammatory biomarkers in hypercholesterolaemic subjects. Br J Nutr. 2011;105(3):417-27. doi:10.1017/S0007114510003697.

13. Mozaffarian D, Clarke R. Quantitative effects on cardiovascular risk factors and coronary heart disease risk of replacing partially hydrogenated vegetable oils with other fats and oils. Eur J Clin Nutr. 2009; 63 Suppl 2:S22-33. doi:10.1038/sj.ejcn.1602976.

14. Alonso A, Ruiz-Gutierrez V, Martínez-González MA. Monounsaturated fatty acids, olive oil and blood pressure: epidemiological, clinical and experimental evidence. Public Health Nutr. 2006;9(2):251-7.

15. Bondia-Pons I, Schröder H, Covas MI, Castellote Al, Kaikkonen J, Poulsen HE, et al. Moderate consumption of olive oil by healthy European men reduces systolic blood pressure in non-Mediterranean participants. J Nutr. 2007;137(1):84-7.

16. Rasmussen BM, Vessby B, Uusitupa M, Berglund L, Pedersen E, Riccardi G, et al. Effects of dietary saturated, monounsaturated, and n-3 fatty acids on blood pressure in healthy subjects. Am J Clin Nutr. 2006;83(2):221-6.

17. Garg A. High-monounsaturated-fat diets for patients with diabetes mellitus: a meta-analysis. Am J Clin Nutr. 1998:67(3 Suppl):577S-82S.

18. Sirtori CR, Tremoli E, Gatti E, Montanari G, Sirtori M, Colli S, et al. Controlled evaluation of fat intake in the Mediterranean diet: comparative activities of olive oil and corn oil on plasma lipids and platelets in high-risk patients. Am J Clin Nutr. 1986;44(5):635-42.

19. Ros E. Dietary cis-monounsaturated fatty acids and metabolic control in type 2 diabetes. Am J Clin Nutr. 2003;78(3 Suppl):617S-25S.

20. Senanayake VK, Pu S, Jenkins DA, Lamarche B, Kris-Etherton PM, West SG et al. Plasma fatty acid changes following consumption of dietary oils containing n-3, n-6, and n-9 fatty acids at different proportions: preliminary findings of the Canola Oil Multicenter Intervention Trial (COMIT). Trials. 2014;15:136. doi:10.1186/1745-6215-15-136.

21. Jones PJ, Senanayake VK, Pu S, Jenkins DJ, Connelly PW, Lamarche B, et al. DHA-enriched high-oleic acid canola oil improves lipid profile and lowers predicted cardiovascular disease risk in the canola oil multicenter randomized controlled trial. Am J Clin Nutr. 2014;100(1):88-97. doi:10.3945/ajen.113.081133.

22. Jones PJ, MacKay DS, Senanayake VK, Pu S, Jenkins DJ, Connelly PW, et al. High-oleic canola oil consumption enriches LDL particle cholesteryl oleate content and reduces LDL proteoglycan binding in humans. Atherosclerosis. 2015;238(2):231-8. doi:10.1016/j.atherosclerosis.2014.12.010.

23. Baril-Gravel L, Labonte ME, Couture P, Vohl MC, Charest A, Guay V, et al. Docosahexaenoic acid-enriched canola oil increases adiponectin concentrations: a randomized crossover controlled intervention trial. Nutr Metab Cardiovasc Dis. 2015;25(1):52-9. doi:10.1016/j.numecd.2014.08.003.

24. Buil-Cosiales P, Zazpe I, Toledo E, Corella D, Salas-Salvadó J, Diez-Espino J, et al. Fiber intake and all-cause mortality in the Prevención con Dieta Mediterránea (PREDIMED) study. Am J Clin Nutr. 2014;100(6):1498-507. doi:10.3945/ajen.114.093757.

25. Cloetens L, Ulmius M, Johansson-Persson A, Akesson B, Onning G. Role of dietary beta-glucans in the prevention of the metabolic syndrome. Nutr Rev. 2012;70(8):444-58

26. Asp NG, Mattsson B, Onning G. Variation in dietary fibre, beta-glucan, starch, protein, fat and hull content of oats grown in Sweden 1987-1989. Eur J Clin Nutr. 1992;46(1):31-7.

27. El Khoury D, Cuda C, Luhovyy BL, Anderson GH. Beta glucan: health benefits in obesity and metabolic syndrome. J Nutr Metab. 2012;2012:851362.

28. U.S. Department of Health and Human Services, Food and Drug Administration. Food labeling: health claims; oats and coronary heart disease. Final rule. 21 CFR part 101. 62(15) Fed Reg. 3584-601. 23 Jan 1997. http://www.gpo.gov/fdsys/pkg/FR-1997-01-23/pdf/97-1598.pdf. Accessed 22 Oct 2015.

29. U.S. Department of Health and Human Services, Food and Drug Administration. Food labeling: health claims; soluble dietary fiber from certain foods and coronary heart disease. Final rule. 70(246) Fed Reg. 76150-62. 23 Dec 2005. http://www.gpo.gov/fdsys/pkg/FR-2005-12-23/pdf/05-24387.pdf. Accessed 22 Oct 2015.

30. European Food Safety Authority (EFSA). EFSA panel on dietetic products, nutrition, and allergies (NDA): scientific opinion on dietary reference values for carbohydrates and dietary fibre. EFSA J. 2010;1462. doi:10.2903/j.efsa.2010.1462.

31. Health Canada, Bureau of Nutritional Sciences, Food Directorate, Health Products and Food Branch. Summary of Health Canada's assessment of a health claim about barley products and blood cholesterol lowering. Ottawa, ON, Canada: Health Canada; July 2012. http://www.hc-sc.gc.ca/fn-an/ 
alt_formats/pdf/label-etiquet/claims-reclam/assess-evalu/barley-orgeeng.pdf. Accessed 22 Oct 2015.

32. Wang Y, Harding SE, Tosh SM, Jones PJ, Ames NP. Barley beta-glucan consumption decreases serum cholesterol level and increases 7 alpha-hydroxy4-cholesten-3-one level in hypercholesterolemic adults [abstract]. FASEB J. 2013;27 Meeting Abstract Supplement:1078.11.

33. Lee JH, O'Keefe JH, Lavie CJ, Marchioli R, Harris WS. Omega-3 fatty acids for cardioprotection. Mayo Clin Proc. 2008:83(3):324-32. doi:10.4065/83.3.324. A published erratum appears in. Mayo Clin Proc. 2008;83(6):730.

34. Kris-Etherton PM, Harris WS, Appel LJ. American Heart Association Nutrition Committee. AHA Scientific Statement: fish consumption, fish oil, omega-3 fatty acids, and cardiovascular disease. Circulation. 2002;106(21):2747-57. A published erratum appears in. Circulation. 2003;107(3):512.

35. Jenkins DJ, Kendall CW, Marchie A, Faulkner DA, Wong JM, de Souza R, et al. Effects of a dietary portfolio of cholesterol-lowering foods vs lovastatin on serum lipids and C-reactive protein. JAMA. 2003;290(4):502-10.

36. Jenkins DJ, Kendall CW, Marchie A, Faulkner DA, Wong JM, de Souza R, et al. Direct comparison of a dietary portfolio of cholesterol-lowering foods with a statin in hypercholesterolemic participants. Am J Clin Nutr. 2005;81 (2):380-7.

37. Theuwissen E, Mensink RP. Simultaneous intake of $\beta$-glucan and plant stanol esters affects lipid metabolism in slightly hypercholesterolemic subjects. J Nutr. 2007;137(3):583-8.

38. Yoshida M, Vanstone CA, Parsons WD, Zawistowski J, Jones PJ. Effect of plant sterols and glucomannan on lipids in individuals with and without type II diabetes. Eur J Clin Nutr. 2006;60(4):529-37.

39. Weidner C, Krempf M, Bard JM, Cazaubiel M, Bell D. Cholesterol lowering effect of a soy drink enriched with plant sterols in a French population with moderate hypercholesterolemia. Lipids Health Dis. 2008;7:35.

40. Mifflin MD, St Jeor ST, Hill LA, Scott BJ, Daugherty SA, Koh YO. A new predictive equation for resting energy expenditure in healthy individuals. Am J Clin Nutr. 1990;51(2):241-7.

41. Wilson PW, D'Agostino RB, Levy D, Belanger AM, Silbershatz H, Kannel WB. Prediction of coronary heart disease using risk factor categories. Circulation. 1998;97(18):1837-47.

42. Friedewald WT, Levy Rl, Fredrickson DS. Estimation of the concentration of low-density lipoprotein cholesterol in plasma, without use of the preparative ultracentrifuge. Clin Chem. 1972;18(6):499-502.

43. Folch J, Lees M, Sloane Stanley GH. A simple method for the isolation and purification of total lipides from animal tissues. J Biol Chem. 1957;226(1):497-509.

44. Jones PJ, Leitch CA, Li ZC, Connor WE. Human cholesterol synthesis measurement using deuterated water: theoretical and procedural considerations. Arterioscler Thromb. 1993;13(2):247-53.

45. Harding SV, Zhao HL, Marinangeli CP, Day AG, Dillon HF, Jain D, et al. Red algal cellular biomass lowers circulating cholesterol concentrations in Syrian golden hamsters consuming hypercholesterolaemic diets. Br J Nutr. 2009;102(6):842-7.

46. Fadrosh DW, Ma B, Gajer P, Sengamalay N, Ott S, Brotman RM, et al. An improved dual-indexing approach for multiplexed 165 rRNA gene sequencing on the Illumina MiSeq platform. Microbiome. 2014;2(1):6 doi:10.1186/2049-2618-2-6

47. Chen J, Bittinger K, Charlson ES, Hoffmann C, Lewis J, Wu GD, et al. Associating microbiome composition with environmental covariates using generalized UniFrac distances. Bioinformatics. 2012;28(16):2106-13. doi:10.1093/bioinformatics/bts342.

\section{Submit your next manuscript to BioMed Central and take full advantage of:}

- Convenient online submission

- Thorough peer review

- No space constraints or color figure charges

- Immediate publication on acceptance

- Inclusion in PubMed, CAS, Scopus and Google Scholar

- Research which is freely available for redistribution 